\title{
Estado epiléptico no convulsivo en el siglo XXI: clínica, diagnóstico, tratamiento y pronóstico
}

\author{
Asier Gómez-Ibáñez, Elena Urrestarazu, César Viteri
}

Resumen. El estado epiléptico no convulsivo es una patología importante para el neurólogo porque, a pesar de su baja prevalencia, puede confundirse con otras entidades, con las consiguientes implicaciones terapéuticas y pronósticas. El diagnóstico está basado en cambios clínicos, fundamentalmente del estado mental o nivel de conciencia habitual del paciente, y electroencefalográficos, por lo que el electroencefalograma es la herramienta básica que hemos de utilizar ante la sospecha clínica. Existen tres tipos: generalizado o estado de ausencia, con grafoelementos epileptiformes difusos en el trazado electroencefalográfico; focal, con descargas localizadas en una área cerebral concreta y que pueden no afectar a la conciencia; y sutil, con actividad epileptiforme focal o difusa asociada a poca o ninguna actividad motora tras una crisis tonicoclónica generalizada o un estado convulsivo. El tratamiento consta de benzodiacepinas y fármacos antiepilépticos; los anestésicos están indicados únicamente en el estado sutil y casos graves de estado parcial complejo. El pronóstico depende principalmente de la etiología y el daño cerebral asociado.

Palabras clave. Benzodiacepinas. Conciencia. Electroencefalograma. Estado no convulsivo.

\section{Introducción}

El estado epiléptico no convulsivo (EENC) es en el siglo XXI uno de los retos más interesantes para los neurólogos clínicos, ya que el retraso diagnóstico y terapéutico repercute en la morbimortalidad de los pacientes.

El término 'estado epiléptico' fue descrito a principios del siglo XIX por médicos ingleses y franceses con diferentes nombres, como furor epilepticus o epileptic mania [1]. A partir de 1929, con el electroencefalograma (EEG) comenzaron a diferenciarse subtipos según etiología, clínica, tratamiento y pronóstico. En 1931, Jackson describió estados prolongados de 'fuga' asociados a la epilepsia [2]. En 1945, Lennox definió el estado de ausencia [3] y Gastaut, en 1956, definió los estados de 'fuga' como fenómenos epilépticos [4], describiendo el estado epiléptico parcial complejo (EEPC). En 1965, Niedermeyer y Khalifeh identificaron como EENC casos de 'estupor con punta-onda' [5]. El último cambio significativo lo introdujo Treiman en 1984, con el concepto de 'estado epiléptico convulsivo generalizado sutil' [6].

Nuestro objetivo es revisar la definición, epidemiología, clínica, diagnóstico, tratamiento y pronóstico del EENC, haciendo especial referencia a las peculiaridades de los distintos subtipos.

\section{Definición}

Hoy en día no hay una definición unitaria para el EENC. En 2007, Meierkord y Holtkamp hablaron de 'cambio en el comportamiento o estado mental respecto del estado basal asociado con descargas epileptiformes continuas en el EEG' [7], y en 2008 Maganti et al lo definieron como 'condición con estado prolongado de alteración del nivel de conciencia asociado a actividad paroxística continua o descargas eléctricas en el EEG' [8]. Estas definiciones recogen los tres aspectos clave: cambios clínicos, electroencefalográficos y simultaneidad de ambos; los dos últimos permiten diferenciarlo de entidades no epilépticas con manifestaciones clínicas similares (Tabla I). Sin embargo, excluyen tipos de EENC, como los focales de origen parietal u occipital, que pueden manifestarse sin alteración de la conciencia, y no definen un patrón en el EEG patognomónico.

Tampoco existe acuerdo en la duración de una crisis para considerarla estado epiléptico. La cifra más consensuada son 30 minutos $[7,9]$, pero algunos autores recomiendan iniciar el tratamiento en el momento del diagnóstico por la arbitrariedad de la cifra [8].

La respuesta electroclínica al tratamiento antiepiléptico ayuda en algunos casos, pero puede darse también en entidades no epilépticas, como la ence-
Unidad de Epilepsia; Departamento de Neurología (A. Gómez-lbáñez, C. Viteri). Unidad de Epilepsia; Servicio de Neurofisiología (E. Urrestarazu). Clínica Universidad de Navarra. Pamplona, Navarra, España.

Correspondencia: Dr. Asier Gómez Ibáñez. Departamento de Neurología. Clínica Universidad de Navarra. Avda. Pío XII, s/n. E-31008 Pamplona (Navarra).

E-mail: agomezi@unav.es

Financiación:

A.G.I. cuenta con una beca predoctoral para formación de investigadores del Gobierno Vasco 2010-2011.

Aceptado tras revisión externa: 05.05.11.

Cómo citar este artículo: Gómez-Ibáñez A, Urrestarazu E, Viteri C. Estado epiléptico no convulsivo en el siglo XXI: clínica, diagnóstico, tratamiento y pronóstico. Rev Neurol 2012; 54: 105-13.

(C) 2012 Revista de Neurología 
Tabla I. Diagnóstico diferencial del estado epiléptico no convulsivo.

Encefalopatía metabólica

Amnesia global transitoria

Amnesia postraumática

Aura migrañosa

Confusión postictal

Intoxicaciones tóxico-farmacológicas

Patologías psiquiátricas

falopatía hepática [10]. Asimismo, podemos encontrar EENC refractarios e incluso desencadenados por los propios fármacos antiepilépticos (FAE) [11].

\section{Epidemiología}

La prevalencia del EENC está infraestimada por la dificultad de su diagnóstico. Aumenta con la edad $[9,12]$ y no hay diferencias entre géneros $[5,13]$. No existen estudios epidemiológicos específicos sobre la incidencia global, pero las estimaciones están entre 2-20 casos por 100.000 habitantes/año [8,14]. El EENC supone entre un 5-49\% del total de los estados epilépticos $[9,15]$; respecto a los diferentes subtipos, hay publicadas prevalencias del $16-43 \%$ en el EEPC $[9,16]$ y del 1-6\% para el estado generalizado o de ausencia (EA) $[9,15]$. En pacientes en coma, la prevalencia es del 8-37\% $[13,17]$.

\section{Clasificación clínica}

\section{Generalidades}

Según los cambios ictales en el EEG, los subtipos más importantes son el generalizado o de ausencia y el focal. Dentro del generalizado, nos encontramos con el típico, atípico y tardío o de novo $[8,18]$; respecto a los focales, tenemos el estado epiléptico parcial simple (EEPS), sin afectación del nivel de conciencia y el EEPC, con disminución de ésta. En cuanto al EENC sutil, no hay acuerdo: unos autores lo clasifican como focal, porque la mayoría de los pacientes presenta una lesión cerebral [8] y descargas laterali- zadas en el EEG [19]; y otros lo clasifican como grupo diferenciado, por su contexto clínico y la presencia de descargas lateralizadas y generalizadas en el EEG [7].

Esta distinción es meramente académica, ya que un mismo episodio de EENC puede adoptar características clínicas y eléctricas diferentes en su evolución. Además, dentro del mismo grupo puede haber diferentes presentaciones electroencefalográficas: en el EEPC del lóbulo frontal, aparecen descargas epileptiformes focales, pero también generalizadas, debido a un fenómeno de sincronización [18].

Las características clínicas y electroencefalográficas de cada subtipo de EENC las describimos a continuación y las resumimos en la tabla II.

\section{Estado epiléptico no convulsivo generalizado o de ausencia}

\section{Estado de ausencia típico}

La característica clínica más importante es la alteración del nivel de conciencia en diferentes grados, de la inatención al estupor [18]. Predominan los cambios conductuales, asociando en ocasiones inexpresividad facial e inestabilidad de la marcha. Algunos pacientes son capaces de realizar actividades complejas y pueden responder alguna orden [20,21]. Hasta en el 50\% de los casos aparecen signos motores como automatismos, parpadeo rítmico, mioclonías, atonía y clonías de predominio facial [20, 22]. La duración es variable, desde minutos (lo habitual) hasta semanas $[23,24]$. Pueden añadirse crisis convulsivas generalizadas al final del episodio [7, $8,18,22]$.

El EEG muestra cualquier actividad epileptiforme continua [25], característicamente punta-onda o polipunta-onda generalizada a 3-4 Hz [18]. En estadios avanzados, los cambios son irregulares y las ondas se lentifican, pudiendo aparecer complejos onda aguda-onda lenta de predominio frontocentral [26]. Las anomalías eléctricas pueden permanecer una vez finalizado el episodio sintomático [27].

El contexto clínico habitual es un paciente con epilepsia generalizada idiopática, bien epilepsia tipo ausencia o epilepsia mioclónica juvenil [24,28]. La fisiopatología parece tener relación con mecanismos gabérgicos y canales de calcio tipo $\mathrm{T}$ talámicos, dentro de la red corticotalamocortical [7]. Los desencadenantes típicos son: insomnio, falta de adhesión a la medicación, menstruación, fiebre, fotoestimulación, hipoglucemia e hiperventilación [22]; asimismo, fármacos como la carbamacepina [29], fenitoína, vigabatrina o tiagabina [22] también pueden provocarlo. 
Tabla II. Características clínicas y electroencefalográficas de los diferentes subtipos de estado epiléptico no convulsivo (adaptado de [7]).

\begin{tabular}{|c|c|c|}
\hline & Manifestaciones clínicas & Manifestaciones en el electroencefalograma \\
\hline $\begin{array}{l}\text { Estado generalizado } \\
\text { o ausencia }\end{array}$ & $\begin{array}{l}\text { Disminución del nivel de conciencia, cambios conductuales, } \\
\text { alucinaciones, parpadeo rítmico, mioclonías sutiles }\end{array}$ & Punta-onda a 2-3 Hz \\
\hline Estado de ausencia típico & Comienzo y fin bruscos, corta duración & Actividad interictal normal \\
\hline Estado de ausencia atípico & $\begin{array}{l}\text { Comienzo y fin mal delimitados, trastornos motores } \\
\text { más evidentes, mayor alteración de la conciencia }\end{array}$ & Actividad interictal con lentificación de fondo \\
\hline Estado de ausencia de novo & Leve amnesia o estupor en pacientes ancianos & Punta-onda a 0,5-4 Hz \\
\hline Estado parcial simple & $\begin{array}{l}\text { Nivel de conciencia conservado, síntomas focales variados } \\
\text { (auditivos, olfatorios, visuales, gustatorios, disestésicos, } \\
\text { psíquicos, vegetativos, conductuales, afasia) }\end{array}$ & $\begin{array}{l}\text { Actividad normal (más frecuente) } \\
\text { Punta o punta-onda focal }\end{array}$ \\
\hline Estado parcial complejo & $\begin{array}{l}\text { Disminución del nivel de conciencia con trastornos } \\
\text { conductuales, automatismos orales o manuales }\end{array}$ & $\begin{array}{l}\text { Actividad normal } \\
\text { Punta o punta-onda focal, } \\
\text { más difusa que en el parcial simple }\end{array}$ \\
\hline Estado sutil & $\begin{array}{l}\text { Estado de coma tras estado convulsivo con/sin movimientos } \\
\text { sutiles en la cara, dedos de las manos o pies, desviación } \\
\text { ocular tónica, nistagmo }\end{array}$ & $\begin{array}{l}\text { Punta o punta-onda lateralizada } \\
\text { o generalizada, patrones periódicos }\end{array}$ \\
\hline
\end{tabular}

\section{Estado de ausencia atípico}

Aparece en pacientes con retraso mental en el contexto de encefalopatías epilépticas como el síndrome de Lennox-Gastaut [30], sugiriéndose una relación directa con el retraso mental [31]. Semiológicamente es parecido al EA típico, pero con tres características propias: mayor intensidad de la alteración del nivel de conciencia, mayor elocuencia de los signos motores y dificultad para delimitar el episodio [18]. Es importante el testimonio de los padres, ya que notarán diferencias respecto del estado basal del niño.

El EEG ictal muestra descargas generalizadas continuas de complejos punta-onda lenta a $2-2,5 \mathrm{~Hz}$ durante al menos el 55\% del registro [18]. En estos casos, es más útil el EEG interictal, que suele estar alterado. De nuevo, FAE como carbamacepina, gabapentina, fenitoína y vigabatrina pueden desencadenar los episodios [29].

\section{Estado de ausencia de novo o tardío}

El dato clínico clave es la edad de los pacientes, fundamentalmente ancianos, por lo que entra en el diagnóstico diferencial del síndrome confusional $[32,33]$. Habitualmente no hay historia de epilepsia. Predomina en las mujeres [33]. Consiste en una alteración del nivel de conciencia, con confusión del paciente. Acompañándolo, a veces encontramos mioclonías faciales e incontinencia esfinteriana [18]. El
EEG es inespecífico, con descargas generalizadas tipo punta-onda irregulares a $0,5-4 \mathrm{~Hz}$ [32]. Los desencadenantes son la retirada brusca de medicación psicotrópica, alteraciones metabólicas o abstinencia alcohólica [34]. El conocimiento de esta entidad evita diagnósticos erróneos de trastornos psiquiátricos en pacientes mayores con cuadros confusionales.

\section{Estado epiléptico no convulsivo focal}

\section{Estado epiléptico parcial simple}

Éste es el único EENC que no presenta alteración del nivel de conciencia. El diagnóstico es difícil por su baja frecuencia [35] y elevado componente subjetivo [36]. Las manifestaciones clínicas van desde fenómenos acústicos, gustatorios u olfatorios, hasta psíquicos, conductuales, vegetativos y visuales, encontrándose también disestesias [8], hemiparesia (EEPS inhibitorio) [36], afasia [37] y piloerección [38]. Estas manifestaciones dependen de la región cortical donde se encuentre o propague el foco epiléptico. El EEG puede ser normal o mostrar descargas epileptiformes focales [8]. Los episodios aparecen en pacientes con lesiones focales cerebrales que hay que buscar por neuroimagen.

\section{Estado epiléptico parcial complejo}

El EEPC se manifiesta de diversas maneras, pero el denominador común es la desconexión del medio 
[39]. Predomina en adultos jóvenes con antecedentes de epilepsia focal, desencadenado por incumplimiento terapéutico, menstruación, estrés o alcohol. Los episodios pueden comenzar con auras prolongadas y asocian habitualmente signos motores semejantes a las crisis parciales complejas (automatismos, versión cefálica o posturas tónicas). Responden de forma heterogénea al tratamiento y tienden a recurrir. La presentación clínica depende del origen. Los temporales cursan con desconexión del medio y automatismos. Los extratemporales más estudiados son los frontales, que se clasifican en tipo I (desinhibición e indiferencia afectiva con leve alteración de conciencia) y tipo II (trastornos conductuales asociados a mayor alteración de conciencia) [40].

El EEPC puede ser cíclico, si aparecen varias crisis aisladas con recuperación parcial de conciencia entre ellas, o continuo, sin recuperación del nivel de conciencia [41].

El patrón del EEG más frecuente es una actividad focal que generaliza rápidamente.

\section{Estado epiléptico no convulsivo sutil}

Este tipo de EENC expresa el agotamiento de los fenómenos motores más evidentes tras un tratamiento inadecuado de un estado epiléptico convulsivo (EEC) [6], aunque también se ha descrito en pacientes sin antecedentes de éste [42]. Los pacientes están en coma, por lo que debemos diferenciarlo de un estado confusional postictal. Para ello nos pueden ayudar signos motores menores, como clonías faciales, parpadeo o movimientos oculares nistagmoides. Pero el diagnóstico definitivo nos lo dará el EEG, con descargas epileptiformes continuas generalizadas que pueden lateralizarse durante la evolución del episodio [6].

\section{Formas atípicas de estado epiléptico no convulsivo}

Estado epiléptico eléctrico durante el sueño lento [22] Consiste en descargas punta-onda generalizadas a 1-3 Hz en más del $85 \%$ del trazado de sueño lento en un EEG de niños entre 3 y 5 años con una epilepsia generalizada grave, aunque a veces también aparece en niños con una epilepsia focal benigna. No produce ningún signo externo o síntoma evidente. Aparece en el 0,5\% de los niños epilépticos y suele desaparecer en la adolescencia.

\section{Estado epiléptico autonómico [43]}

Aparece en pacientes con el síndrome de Panayiotopoulos. Clínicamente se manifiesta con náuseas, vómitos, desviación ocular, incontinencia urinaria, palidez mucocutánea, hiperventilación o cefalea. No hay alteración del nivel de conciencia. En el EEG destacan descargas tipo punta-onda a $3 \mathrm{~Hz}$ occipitales, con frecuente fotosensibilidad. Puede aparecer hasta en el $6 \%$ de niños epilépticos y el pronóstico es bueno, no requiriendo tratamiento específico en su mayoría porque no suelen recurrir.

\section{Estado epiléptico no convulsivo en patología no epiléptica}

El 70\% de los mayores de 60 años que presentan EENC no tiene una epilepsia conocida, proporción que se invierte a edades más tempranas [15]. Merecen atención especial los pacientes en coma, resultado final común de múltiples patologías.

\section{Enfermedades neurológicas no epilépticas}

Las lesiones agudas cerebrales, tanto focales como difusas, representan una causa frecuente de EENC porque alteran la arquitectura de las neuronas corticales y de sus conexiones. Las focales más frecuente son las vasculares y tumorales (primarias o metástasicas) [7], pero otras, como las lesiones desmielinizantes, también desencadenan el EENC [44]. Las difusas más importantes son las encefalopatías hipoxicoisquémica e hipertensiva, el síndrome de encefalopatía posterior reversible, infecciones como meningitis, encefalitis, neurosífilis o enfermedad de CreutzfeldtJakob, y patologías neurooncológicas como carcinomatosis meníngea o síndromes paraneoplásicos $[8$, 33]. Asimismo, hay descritos EENC tras la administración de terapia electroconvulsiva [45].

\section{Enfermedades y situaciones sistémicas}

Destacan las alteraciones metabólicas (hipo e hiperglucemia, hipocalcemia, hiponatremia, hiperamoniemia, uremia e hipertiroidismo), la porfiria aguda y los síndromes serotoninérgico y neuroléptico maligno. Además, hay descritos casos con fármacos, como antibióticos (cefalosporinas, carbapeménicos, quinolonas, isoniacida), inmunosupresores (ciclosporinas y tacrolimus), quimioterápicos (ifosfamida), psicotrópicos (antidepresivos tricíclicos, olanzapina, litio) y FAE (carbamacepina, vigabatrina, fenitoína o tiagabina). También se han visto involucradas drogas de abuso (cocaína, anfetaminas, heroína) [7]. Pacientes con enfermedades autoinmunes sistémicas con afectación del sistema nervioso central, como lupus eritematoso o púrpura trombótica trombocitopénica, han desarrollado cuadros estuporosos identificados como EENC [46,47]. 


\section{Estado de coma}

La principal duda en pacientes en coma es si su situación se debe a un EENC o a la disfunción neurológica subyacente. El origen epiléptico se sospechará cuando el paciente ha presentado previamente un EEC, tiene historia previa de crisis epilépticas o en el EEG aparece actividad rítmica sugestiva de crisis electroencefalográfica. Sin embargo, la interpretación de los hallazgos electroencefalográficos es controvertida ya que, en fases terminales de procesos con daño cerebral difuso, aparecen anomalías epileptiformes continuas o periódicas [48], no necesariamente responsables del coma, sino probablemente epifenómenos eléctricos causados por la disfunción cerebral subyacente [49]. El EENC en pacientes en coma tiene en sí mismo un valor pronóstico [50], aunque éste depende fundamentalmente de la etiología del coma.

\section{Diagnóstico}

La historia clínica y exploración física pueden ser indistinguibles de otras entidades en ocasiones, pero hay que buscar datos sugestivos. La combinación de factores de riesgo para desarrollar un foco epileptógeno (ictus, neurocirugía, tumor, meningoencefalitis), alteraciones oculomotoras (nistagmo, parpadeo rítmico, desviación ocular mantenida) o sudoración puede llegar a una sensibilidad diagnóstica cercana al 100\% [51]. La especificidad es más baja, confirmándose el diagnóstico mediante EEG entre el 45$68 \%$ de los pacientes en los que se sospecha $[52,53]$. Por tanto, la piedra angular en el diagnóstico tanto de crisis no convulsivas como de EENC es el EEG y/o la monitorización electroencefalográfica continua [54], mostrando actividad epileptiforme coincidiendo con la clínica. La limitación más importante es la ausencia de un patrón patognomónico y la presencia de grafoelementos, como las descargas periódicas, en que no hay acuerdo sobre su origen, ya que algunos autores lo consideran un patrón interictal [55]. Estas descargas periódicas aparecen en encefalopatías toxicometabólicas o anoxoisquémicas, enfermedades neurodegenerativas o infecciones del sistema nervioso central, y pueden ser lateralizadas, lateralizadas bilaterales independientes, generalizadas, ondas trifásicas e inducidas por un estímulo [56]. Recientemente, se ha descrito la utilidad, asimismo, de la tomografía por emisión de positrones con fluorodeoxiglucosa en el diagnóstico de un EEPC [57].

En definitiva, para el diagnóstico de EENC es necesaria una valoración conjunta del contexto clí- nico y el EEG. Desde el punto de vista opuesto, para considerar que ha cedido el episodio, es imprescindible la constatación de una mejoría clínica asociada al cese de la actividad electroencefalográfica [11].

\section{Tratamiento}

Los objetivos del tratamiento son, a corto plazo, el cese de las descargas epilépticas y la mejoría clínica $y$, a largo plazo, la prevención del daño cerebral secundario a la actividad epileptiforme. Muchos pacientes asocian un trastorno del nivel de conciencia, por lo que el primer paso es mantener un soporte vital básico (preservación de vía aérea, monitorización electrocardiográfica, canulación de vía periférica y control de la presión arterial y saturación de oxígeno). Un segundo escalón nos llevará a la identificación de la causa del EENC para poder asociar un tratamiento etiológico específico, por ejemplo, antibióticos en meningitis.

No hay actualmente evidencia científica de ensayos clínicos aleatorizados sobre un tratamiento establecido para el EENC, por lo que las recomendaciones están basadas en la experiencia clínica y estudios con pocos sujetos. Tampoco existen estudios comparativos entre los fármacos. La variabilidad clínica hace que las opciones terapéuticas y la intensidad con la que son aplicadas sean diversas, debiendo aplicar un tratamiento individualizado para cada paciente, según el subtipo clínico, la etiología y su estado general.

De forma académica, hemos diferenciado dos grupos: pacientes fuera del estado de coma y pacientes en coma.

\section{Pacientes fuera del estado de coma}

Los fármacos de primera elección son las benzodiacepinas: loracepam $(0,1 \mathrm{mg} / \mathrm{kg}$ con infusión a $2 \mathrm{mg} / \mathrm{min})$ $[11,58]$, clobazam [59], clonacepam (1 mg) o diacepam (10 mg) [60]. La vía de administración es oral o intravenosa [60]. En ancianos hay más riesgo de efectos adversos cardiorrespiratorios, por lo que algunos autores recomiendan comenzar directamente con ácido valproico [61]. Los EA de novo responden bien y sin recurrencias; los EA típicos responden, pero recurren con frecuencia; y los EA atípicos y EENC focales suelen ser refractarios [8]. Excepto en el EA por privación de fármacos o alcohol, debemos reajustar el tratamiento de base con FAE.

El segundo paso en un EENC refractario es administrar un FAE endovenoso. Los clásicos son ácido valproico (bolo de 25-45 mg/kg con infusión con- 


\begin{tabular}{ll}
$\begin{array}{l}\text { Tabla III. Esquema terapéutico general del estado epiléptico no convul- } \\
\text { sivo en pacientes que no están en coma }\end{array}$ \\
\hline Estado de ausencia típico & $\begin{array}{l}\text { Benzodiacepinas } \\
\text { Antiepilépticos (excepto fenitoína) }\end{array}$ \\
\hline Estado de ausencia atípico & $\begin{array}{l}\text { Benzodiacepinas } \\
\text { Antiepilépticos (excepto fenitoína) }\end{array}$ \\
\hline Estado de & $\begin{array}{l}\text { Benzodiacepinas } \\
\text { antiepiléptico (excepto pacientes } \\
\text { con retirada brusca de fármacos } \\
\text { o abstinencia alcohólica) }\end{array}$ \\
\hline Estado parcial simple & $\begin{array}{l}\text { Benzodiacepinas } \\
\text { Antiepilépticos }\end{array}$ \\
\hline Estado parcial complejo & $\begin{array}{l}\text { Benzodiacepinas } \\
\text { Antiepilépticos } \\
\text { Anestésicos (en casos seleccionados) }\end{array}$ \\
\hline Estado sutil & $\begin{array}{l}\text { Benzodiacepinas } \\
\text { Antiepilépticos } \\
\text { Anestésicos }\end{array}$ \\
\hline
\end{tabular}

tinua de $1 \mathrm{mg} / \mathrm{kg} / \mathrm{h}$ ) para todos los tipos de EENC, así como fenitoína (bolo de $15-20 \mathrm{mg} / \mathrm{kg}$ con infusión continua a un máximo de $50 \mathrm{mg} / \mathrm{min}$, hasta 30 $\mathrm{mg} / \mathrm{kg} /$ día) y fenobarbital (18 mg/kg) sólo para los focales, ya que fenitoína y fenobarbital pueden agravar los EA [11,60]. Una cuarta opción es la fosfenitoína, mejor tolerada que la fenitoína, pero con poca experiencia de uso [11]. El ácido valproico es de primera elección, porque abarca más subtipos clínicos, tiene mayor tolerabilidad, menos efectos adversos y el manejo es más sencillo, ya que no requiere monitorización electrocardiográfica [11]. No obstante, puede producir interacciones con fenobarbital o lamotrigina [11]. Asimismo, hay casos descritos de respuesta con levetiracetam (bolos de $1.000 \mathrm{mg}$ con dosis medias de $2.000 \mathrm{mg} /$ día) [62], topiramato [63] y lacosamida [64].

El tratamiento con anestésicos no está indicado, en general, en el EENC cuando el paciente no está en coma. La única excepción sería un paciente joven, sin otra patología y refractario a dos o tres FAE [8]. En este caso, las opciones son parecidas a las de un EEC: tiopental (bolo de 3-5 mg/kg más bolo de 1-2 mg/kg cada 2-3 minutos hasta el control de la crisis, con infusión a 3-7 mg/kg/h), midazolam (bolo de $0,2 \mathrm{mg} / \mathrm{kg}$ más infusión a 0,05-0,4 $\mathrm{mg} / \mathrm{kg} / \mathrm{h}$ ) y propofol (bolo de $2-3 \mathrm{mg} / \mathrm{kg}$ más bolo de $1-2 \mathrm{mg} / \mathrm{kg}$ hasta el control de la crisis, con infusión a 4-10 mg/ $\mathrm{kg} / \mathrm{h}$ ) [60]. El propofol puede ocasionar un fallo multiorgánico debido a la inhibición de la producción de energía intracelular, conocido como PRIS (propofol infusion syndrome) [65]. Asimismo, hay casos descritos de resolución de EENC focales mediante transecciones subpiales [66] o hipotermia a $30-31{ }^{\circ} \mathrm{C}$ asociada a diacepam en casos refractarios a anesté$\operatorname{sicos}[67]$.

En la tabla III indicamos un esquema terapéutico del EENC en pacientes no comatosos.

\section{Pacientes en coma}

\section{Coma tras estado epiléptico convulsivo- estado epiléptico no convulsivo sutil}

Estos pacientes tienen mejor pronóstico porque no asocian otra enfermedad sistémica, por lo que el coma se resuelve con el tratamiento del propio estado epiléptico. La respuesta a benzodiacepinas y FAE oscila entre el 8-24\% [8], por lo que la mayoría requiere anestésicos según el esquema previo [11, $58,60]$. La hipotermia podría ser, de nuevo, una opción en casos refractarios [67].

\section{Coma secundario a otras patologías}

Este grupo requiere menor agresividad terapéutica, porque las alteraciones en el EEG reflejan el daño cerebral causante del coma y el objetivo prioritario debe ser, por tanto, el tratamiento de la etiología. El pronóstico en general es malo y depende de la etiología, de la profundidad del coma y del desarrollo de complicaciones agudas. En general, las recomendaciones son tratar con una benzodiacepina; si mejora, comenzamos con FAE, por ejemplo, ácido valproico; y si no mejora, tratamiento de soporte [11]. Hay que tener en cuenta, no obstante, que la mayoría de estos pacientes están con anestésicos, que ya poseen un efecto antiepiléptico por sí mismos.

\section{Daño cerebral}

Permanece sin resolver aún la cuestión acerca del daño en términos de pérdida neuronal que el EENC pueda provocar en el cerebro humano; en todo caso, éste será probablemente diferente en los distintos subtipos.

En modelos animales de EEPC, hay pérdida neuronal en el hipocampo con sprouting de fibras musgosas, similar a la epilepsia del lóbulo temporal [68], y en estructuras extrahipocampales [69]. Los cambios se relacionan con excitotoxicidad por activación de receptores glutamatérgicos NMDA [70], flujo de calcio iónico [71] o reactivos de oxígeno y nitrógeno, con disfunción mitocondrial como resultado final $[72,73]$. 
En humanos, en cambio, no se ha encontrado una clara reducción de la densidad neuronal [74]. Estudios con marcadores indirectos de daño neuronal (enolasa específica neuronal en el líquido cefalorraquídeo y resonancia magnética con espectroscopia) han objetivado alguna alteración [75, 76], pero las consecuencias clínicas de estos hallazgos son motivo de controversia. No hay estudios prospectivos, e incluso uno transversal no encontró diferencias en la función neuropsicológica global de pacientes epilépticos con y sin episodios de EENC [77].

\section{Pronóstico}

Todos los subtipos de EENC, excepto el sutil, son benignos por sí mismos en términos de morbimortalidad. El dato más importante para el pronóstico es la causa que ha provocado el EENC, especialmente en los pacientes mayores en coma [78]. La mortalidad en pacientes con EENC y antecedentes de epilepsia es alrededor de un $3 \%$, mientras que la mortalidad de pacientes con EENC secundario a patología aguda es de un 27\% [79]; la mortalidad en pacientes en coma está entre el 47-60\%, parecida a pacientes en coma sin descargas epileptiformes, que varía entre el 54-66\% [13,80].

\section{Conclusiones}

El EENC comprende un amplio espectro de situaciones clínicas englobadas bajo las características comunes de cambios clínicos, la mayoría alteraciones del nivel de conciencia o conductuales, asociados a cambios electroencefalográficos epileptiformes. Por tanto, la herramienta básica diagnóstica es el EEG. Debido a su baja prevalencia y expresividad clínica, puede pasar fácilmente inadvertido y ser confundido con otras patologías, especialmente psiquiátricas.

El EENC puede ser la manifestación de un daño cerebral o sistémico agudo que requiera tratamiento urgente, siendo esto especialmente relevante en pacientes en coma, ya que la causa va a ser el factor pronóstico más importante. Los distintos subtipos de EENC producen diferente grado de daño neuronal porque, aunque requiere un estudio individualizado del paciente, en líneas generales el tratamiento depende del subtipo. En la mayoría de los casos el tratamiento es conservador, pero algunos pueden requerir tratamientos agresivos, tanto del EENC como de su causa.

\section{Bibliografía}

1. Kaplan PW. The clinical features, diagnosis, and prognosis of nonconvulsive status epilepticus. Neurologist 2005; 11: 348-61.

2. Jackson JH. Epilepsy and epileptiform convulsions. In Taylor J, ed. Selected writings of John Hughlings Jackson. Vol. 1. London: Hodder \& Stoughton; 1931. p. 1-7.

3. Lennox WG. The treatment of epilepsy. Med Clin N Am 1945; 29: 1114-28.

4. Gastaut H, Roger J, Roger A. Sur la signification de certaines fugues épileptiques: états de mal temporal. Rev Neurol (Paris) 1956; 94: 298-301.

5. Niedermeyer E, Khalifeh R. Petit mal status ('spike-wave stupor'): an electro-clinical appraisal. Epilepsia 1965; 6: 250-62.

6. Treiman DM, DeGiorgio CMA, Salisbury SM, Wickboldt CL Subtle generalized convulsive status epilepticus. Epilepsia 1984; 25: 653.

7. Meierkord H, Holtkamp M. Non-convulsive status epilepticus in adults: clinical forms and treatment. Lancet 2007; 6: 329-39.

8. Maganti R, Gerber P, Drees C, Chung S. Nonconvulsive status epilepticus. Epilepsy Behav 2008; 12: 572-86.

9. Knake S, Rosenow F, Vescovi M, Oertel WH, Mueller HH, Wirbatz A, et al. Incidence of status epilepticus in adults in Germany: a prospective, population-based study. Epilepsia 2001; 42: 714-8.

10. Kaplan PW. Prognosis in nonconvulsive status epilepticus. Epileptic Disord 2000; 2: 185-93.

11. Van Ruckevorsel K, Boon P, Hauman H, Legros B, Ossemann M, Sadzot B, et al. Standards of care for non-convulsive status epilepticus: Belgian consensus recommendations. Acta Neurol Belg 2006; 106: 117-24.

12. Commission on Classification and Terminology of the International League Against Epilepsy. Proposal for revised clinical and electroencephalographic classification of epileptic seizures. Epilepsia 1981; 22: 489-501.

13. Towne AR, Waterhouse EJ, Boggs JG, Garnett LK, Brown AJ, Smith JR Jr, et al. Prevalence of nonconvulsive status epilepticus in comatose patients. Neurology 2000; 54: 340-9.

14. Walker M, Cross H, Smith S, Young C, Aicardi J, Appleton R, et al. Nonconvulsive status epilepticus: Epilepsy Research Foundation Workshop Reports. Epileptic Disord 2005; 7: 253-96.

15. DeLorenzo RJ, Hauser WA, Towne AR, Boggs JG, Pellock JM, Penberthy L, et al. A prospective, population-based epidemiologic study of status epilepticus in Richmond, Virginia. Neurology 1996; 46: 1029-35.

16. Vignatelli L, Tonon C, D'Alessandro R. Incidence and shortterm prognosis of status epilepticus in adults in Bologna, Italy. Epilepsia 2003; 44: 964-8.

17. Privitera MD, Strawsburg R. Management of seizures in the emergency department. In Jagoda A, Riggio S, eds. Emergency medicine clinics of North America. Vol. 12. Philadelphia: W.B. Saunders; 1994. p. 1089-100.

18. Fernández-Torre JL, Gutiérrez-Pérez R, Velasco-Zarzosa M. Estado epiléptico no convulsivo. Rev Neurol 2003; 37: 744-52.

19. Treiman DM, Walton NY, Kendrick C. A progressive sequence of electroencephalographic changes during generalized convulsive status epilepticus. Epilepsy Res 1990; 5: 49-60.

20. Andermann F, Robb JP. Absence status: a reappraisal following review of thirty-eight patients. Epilepsia 1972; 13: 177-87.

21. Drislane FW. Presentation, evaluation, and treatment of nonconvulsive status epilepticus. Epilepsy Behav 2000; 1: 301-14.

22. Shorvon S, Walker M. Status epilepticus in idiopathic generalized epilepsy. Epilepsia 2005; 46 (Suppl 9): S73-9.

23. Cascino GD. Nonconvulsive status epilepticus in adults and children. Epilepsia 1993; 34 (Suppl 1): S21-8.

24. Agathonikou A, Panayiotopoulos CP, Giannakodimos S. Typical absence status in adults: diagnostic and syndromic considerations. Epilepsia 1998; 39: 1265-76.

25. Porter RJ, Penry JK. Petit mal status. In Delgado-Escueta AV, Wasterlain CG, Treiman DM, Porter RJ, eds. Advances in Neurology. Vol. 34. Status epilepticus. New York: Raven Press; 1983. p. 61-7. 
26. Treiman DM. Electroclinical features of status epilepticus. J Clin Neurophysiol 1995; 12: 343-62.

27. Gökyigit A, Çaliskan A. Diffuse spike-wave status of 9-year duration without behavioral change or intellectual decline. Epilepsia 1995; 36: 210-3.

28. Tomson T, Lindbom U, Nilsson BY. Nonconvulsive status epilepticus in adults: thirty-two consecutive patients from a general hospital population. Epilepsia 1992; 33: 829-35.

29. Thomas P, Valton L, Genton P. Absence and myoclonic status epilepticus precipitated by antiepileptic drugs in idiopathic generalized epilepsy. Brain 2006; 129: 1281-92.

30. Snead OC 3rd, Dean JC, Penry JK. Absence status epilepticus. In Engel J Jr, Pedley TA, eds. Epilepsy: a comprehensive textbook. Philadelphia: Lippincott-Raven; 1997. p. 701-77.

31. Hoffmann-Riem M, Diener W, Benninger CHR, Rating D, Unnebrink K, Stephani U, et al. Nonconvulsive status epilepticus -a possible cause of mental retardation in patients with Lennox-Gastaut syndrome. Neuropediatrics 2000; 31: 169-74.

32. Thomas P, Beaumanoir A, Genton P, Dolisi C, Chatel M. 'De novo' absence status of late onset: report of 11 cases. Neurology 1992; 42: 104-10.

33. Fernández-Torre JL. De novo absence status of late onset following withdrawal of lorazepam: a case report. Seizure 2001; 10: 433-7.

34. Thomas P, Lebrun C, Chatel M. De novo absence status epilepticus as a benzodiazepine withdrawal syndrome. Epilepsia 1993; 34: 355-8.

35. Wieser HG. Simple partial status epilepticus. In Engel J Jr, Pedley TA, eds. Epilepsy: a comprehensive textbook. Philadelphia: Lippincott-Raven; 1997. p. 709-23.

36. Armon C, Radtke RA, Friedman AH. Inhibitory simple partial (nonconvulsive) status epilepticus after intracranial surgery. J Neurol Neurosurg Psychiatry 2000; 69: 18-24.

37. Chung PW, Seo DW, Kwon JC, Kim H, Na DL. Nonconvulsive status epilepticus presenting as subacute progressive aphasia. Seizure 2002; 11: 449-54.

38. Roze E, Oubary P, Chédru F. Status-like recurrent pilomotor seizures: case report and review of the literature. J Neurol Neurosurg Psychiatry 2000; 68: 647-9.

39. Salas-Puig J, Suárez-Moro R, Mateos V. Status epilepticus. Neurologia 1996; 11 (Suppl 4): S108-21.

40. Thomas P, Zifkin B, Migneco O, Lebrun C, Darcourt J, Andermann F. Nonconvulsive status epilepticus of frontal origin. Neurology 1999; 52: 1174-83.

41. Williamson PD. Complex partial status epilepticus. In Engel J Jr, Pedley TA, eds. Epilepsy: a comprehensive textbook. Philadelphia: Lippincott-Raven; 1997. p. 681-99.

42. Costello DJ, Cole AJ. Treatment of acute seizures and status epilepticus. J Intensive Care Med 2007; 22: 319-47.

43. Panayiotopoulos CP. Autonomic seizures and autonomic status epilepticus peculiar to childhood: diagnosis and management. Epilepsy Behav 2004; 5: 286-95.

44. Trinka E, Unterberger I, Spiegel M, Niedermüller U, Benke T, Berger T, et al. De novo aphasic status epilepticus as presenting symptom of multiple sclerosis. J Neurol 2002; 249: 782-3.

45. Varma NK, Lee SI. Nonconvulsive status epilepticus following electroconvulsive therapy. Neurology 1992; 42: 263-4.

46. Fernández-Torre JL, Sánchez JM, González C, FernándezGuinea O. Complex partial status epilepticus of extratemporal origin in a patient with systemic lupus erythematosus. Seizure 2003; 12 : 245-8.

47. Blum AS, Drislane FW. Nonconvulsive status epilepticus in thrombotic thrombocytopenic purpura. Neurology 1996; 47: 1079-81.

48. Kaplan PW. The EEG of status epilepticus. J Clin Neurophysiol 2006; 23: 221-9.

49. Brenner RP. EEG in convulsive and nonconvulsive status epilepticus. J Clin Neurophysiol 2004; 21: 319-31.

50. Bauer G, Trinka E. Nonconvulsive status epilepticus and coma. Epilepsia 2010; 51: 177-90.

51. Husain AM, Horn GJ, Jacobson MP. Non-convulsive status epilepticus: usefulness of clinical features in selecting patients for urgent EEG. J Neurol Neurosurg Psychiatry 2003; 74: 189-91.

52. Praline J, Grujic J, Corcia P, Lucas B, Hommet C, Autret A, et al. Emergent EEG in clinical practice. Clin Neurophysiol 2007; 118: 2149-55.

53. Lorenzl S, Mayer S, Feddersen B, Jox R, Noachtar S, Borasio GD. Nonconvulsive status epilepticus in palliative care patients. J Pain Symptom Manage 2010; 40: 460-5.

54. Fernández-Torre JL. Estado epiléptico no convulsivo en adultos en coma. Rev Neurol 2010; 50: 300-8.

55. Hughes JR. Periodic lateralized epileptiform discharges: do they represent an ictal pattern requiring treatment? Epilepsy Behav 2010; 18: 162-5.

56. Brenner RP. How useful is EEG monitoring in acutely ill and how to interpret it? Epilepsia 2009; 50 (Suppl 12): S34-7.

57. Stayman A, Abou-Khalil B. FDG-PET in the diagnosis of complex partial status epilepticus originating from the frontal lobe. Epilepsy Behav 2011; 20: 721-4.

58. Meierkord H, Boon P, Engelsen B, Göcke K, Shorvon S, Tinuper $\mathrm{P}$, et al. EFNS guidelines on the management of status epilepticus in adults. Eur J Neurol 2010; 17: 348-55.

59. Corman C, Guberman A, Benavente O. Clobazam in partial status epilepticus. Seizure 1998; 7: 243-7.

60. Walker MC. Treatment of nonconvulsive status epilepticus. Int Rev Neurobiol 2007; 81: 287-97.

61. Sinha S, Naritoku DK. Intravenous valproate is well tolerated in unstable patients with status epilepticus. Neurology 2000, 55: 722-4.

62. Möddel G, Bunten S, Dobis C, Kovac S, Dogan M, Fischera M, et al. Intravenous levetiracetam: a new treatment alternative for refractory status epilepticus. J Neurol Neurosurg Psychiatry 2009; 80: 689-92.

63. Towne AR, Garnett LK, Waterhouse EJ, Morton LD, DeLorenzo RJ. The use of topiramate in refractory status epilepticus. Neurology 2003; 60: 332-4.

64. Kellinghaus C, Berning S, Besselmann M. Intravenous lacosamide as successful treatment for nonconvulsive status epilepticus after failure of first-line therapy. Epilepsy Behav 2009; 14: 429-31.

65. Diedrich DA, Brown DR. Propofol infusion syndrome in the ICU. J Intensive Care Med 2011; 26: 59-72

66. Costello DJ, Simon MV, Eskandar EN, Frosch MP, Henninger $\mathrm{HL}$, Chiappa KH, et al. Efficacy of surgical treatment of de novo, adult-onset, cryptogenic, refractory focal status epilepticus. Arch Neurol 2006; 63: 895-901.

67. Schmitt FC, Buchheim K, Meierkord H, Holtkamp M. Anticonvulsant properties of hypothermia in experimental status epilepticus. Neurobiol Dis 2006; 23: 689-96.

68. Hosford DA. Animal models of nonconvulsive status epilepticus. J Clin Neurophysiol 1999; 16: 306-13.

69. Krsek P, Mikulecka A, Druga R, Kubova H, Suchomelova l, Mares P. Long-term behavioral and morphological consequences of nonconvulsive status epilepticus in rats. Epilepsy Behav 2004; 5: 180-91.

70. Fountain NB, Lothman EW. Pathophysiology of status epilepticus. J Clin Neurophysiol 1995; 12: 326-42.

71. Pal S, Sombati S, Limbrick DD, DeLorenzo RD. In vitro status epilepticus causes sustained elevation of intracellular calcium levels in hippocampal neurons. Brain Res 1999; 851: 20-31.

72. Cock HR. The role of mitochondria and oxidative stress in neuronal damage after brief and prolonged seizures. Prog Brain Res 2002; 135: 187-96.

73. Nehlig A, De Vasconcelos AP. The model of pentylenetetrazolinduced status epilepticus in the immature rat: short- and long-term effects. Epilepsy Res 1996; 26: 93-103.

74. Tsuchida TN, Barkovich AJ, Bollen AW, Hart AP, Ferriero DM. Childhood status epilepticus and excitotoxic neuronal injury. Pediatr Neurol 2007; 36: 253-7.

75. Rabinowicz AL, Correale JD, Bracht KA, Smith TD, DeGiorgio $\mathrm{CM}$. Neuron-specific enolase is increased after nonconvulsive status epilepticus. Epilepsia 1995; 36: 475-9.

76. Lazeyras F, Blanke O, Zimine I, Delavelle J, Perrig SH, Seeck M. MRI, $1 \mathrm{H}-\mathrm{MRS}$, and functional MRI during and after 
prolonged nonconvulsive seizure activity. Neurology 2000; 55: $1677-82$.

77. Adachi N, Kanemoto K, Muramatsu R, Kato M, Akanuma $\mathrm{N}$, Ito $\mathrm{M}$, et al. Intellectual prognosis of status epilepticus in adult epilepsy patients: analysis with Wechsler Adult Intelligence Scale Revised. Epilepsia 2005; 46: 1502-9.

78. Litt B, Wityk RJ, Hertz SH, Mullen PD, Weiss H, Ryan DD, et al. Nonconvulsive status epilepticus in the critically ill elderly. Epilepsia 1998; 39: 1194-202.

79. Shneker BF, Fountain NB. Assessment of acute morbidity and mortality in nonconvulsive status epilepticus. Neurology 2003; 61: 1066-73.

80. Lowenstein DH, Aminoff MJ. Clinical and EEG features of status epilepticus in comatose patients. Neurology 1992; 42: 100-4.

Non-convulsive status epilepticus in the 21st century: clinical characteristics, diagnosis, treatment and prognosis

Summary. Non-convulsive status epilepticus is a significant issue for a neurologist because, despite its low prevalence, it mimics other pathologies, with therapeutics and prognostic outcomes. Diagnosis is based on clinical features, mainly mental status or impaired consciousness and electroencephalographic changes, so electroencephalogram is the first exploration we must perform with clinical suspicion. There are three clinical forms: generalized or absence status, with diffuse epileptiform discharges; focal, with epileptic discharges located in a specific brain area and may not affect consciousness; and subtle, with diffuse or local epileptic activity after a tonic-clonic seizure or convulsive status and limited or no motor activity. Treatment are benzodiazepines and antiepileptic drugs; anesthetic drugs are only recommended for patients with subtle status and in some with partial complex status. Prognosis is mainly determined by etiology and associated brain damage.

Key words. Benzodiazepines. Consciousness. Electroencephalogram. Non-convulsive status. 